DSF-23/2006, IFIC/06-24, MPP-2006-80

\title{
Effects of non-standard neutrino-electron interactions on relic neutrino decoupling
}

\author{
Gianpiero Mangano ${ }^{\mathrm{a}}$, Gennaro Miele ${ }^{\mathrm{a}}$, Sergio Pastor ${ }^{\mathrm{b}}$, \\ Teguayco Pinto $^{b}$, Ofelia Pisanti ${ }^{a}$, Pasquale D. Serpico ${ }^{c}$ \\ ${ }^{a}$ Dipartimento di Scienze Fisiche, Università di Napoli Federico II and INFN, \\ Sezione di Napoli, Complesso Universitario di Monte S. Angelo \\ Via Cintia, I-80126 Naples, Italy \\ ${ }^{\mathrm{b}}$ Instituto de Física Corpuscular (CSIC-Universitat de València), \\ Ed. Institutos de Investigación, Apdo. 22085, E-46071 Valencia, Spain \\ ${ }^{\mathrm{c}}$ Max-Planck-Institut für Physik (Werner-Heisenberg-Institut), \\ Föhringer Ring 6, D-80805 Munich, Germany
}

\begin{abstract}
We consider the decoupling of neutrinos in the early Universe in presence of nonstandard neutral current neutrino-electron interactions (NSI). We first discuss a semi-analytical approach to solve the relevant kinetic equations and then present the results of fully numerical and momentum-dependent calculations, including flavor neutrino oscillations. We present our results in terms of both the effective number of neutrino species $\left(N_{\text {eff }}\right)$ and the impact on the abundance of ${ }^{4} \mathrm{He}$ produced during Big Bang Nucleosynthesis. We find that the presence of neutrino-electron NSI may enhance the entropy transfer from electron-positron pairs into neutrinos instead of photons, up to a value of $N_{\text {eff }} \simeq 3.12$ for NSI parameters within the ranges allowed by present laboratory data, which is almost three times the effect that appears for standard weak interactions. Thus non-standard neutrino-electron interactions do not essentially modify the density of relic neutrinos nor the bounds on neutrino properties from cosmological observables, such as their mass.
\end{abstract}

Key words: Early Universe; Neutrinos; Non-equilibrium kinetics

\section{Introduction}

In the early Universe, neutrinos were kept in thermal contact with the electromagnetic primordial plasma by rapid weak interactions with electrons and positrons. When the temperature dropped below a few $\mathrm{MeV}$, these weak 
processes became ineffective and the process of neutrino decoupling took place, while shortly after the $e^{ \pm}$pairs began to annihilate almost entirely into photons thus producing a difference between the temperatures of the relic photons and neutrinos. This difference can be easily calculated if we assume that neutrinos were completely decoupled when the $e^{ \pm}$pairs transferred their entropy to photons, leading to the well-known temperature ratio $T_{\gamma} / T_{\nu}=(11 / 4)^{1 / 3} \simeq 1.40102$. Indeed, this simplified picture should be improved since some relic interactions between $e^{ \pm}$and neutrinos exist all along the $e^{ \pm}$annihilation stage, leading to a slightly smaller increase of the comoving photon temperature and to small distortions (at the percent level) of the neutrino momentum distributions.

Presently, there exist compelling evidences for flavor neutrino oscillations from a variety of experimental data on solar, atmospheric, reactor and accelerator neutrinos (see e.g. $[1,2]$ ). These results are well understood by assuming that neutrinos have masses and mix, which in turn seems to point out the necessity of some new physics beyond the Standard Model (SM) of fundamental interactions. Interestingly, non-zero neutrino masses usually come with nonstandard interactions (NSI) that might violate leptonic flavor and/or break weak universality. Recent analyses $[3,4,5,6]$ have considered the neutral current NSI in a phenomenological way, showing that they can be bound using measurements of neutrino-electron scattering, as well as data from LEP and from related charged lepton processes.

The aim of this paper is to study the neutrino decoupling process in presence of additional interactions between neutrinos and electrons, a possibility already noted in $[3,5]$. In this case, neutrinos could be kept in longer contact with $e^{ \pm}$and thus share a larger amount of the total entropy transfer than in the SM. Actually, if the non-standard neutrino-electron interactions were large enough, the neutrino momentum distribution would be significantly different from the standard case. In turn, this would modify the final yield of light nuclei during the epoch of Big Bang Nucleosynthesis (BBN), as well as the radiation content of the Universe, affecting the anisotropies of the Cosmic Microwave Background (CMB) and the power spectrum of Large Scale Structures (LSS). Our goal is to calculate how the decoupling is modified taking into account NSI with couplings which are still allowed by present laboratory data, and to discuss the possibility that cosmological observations can be used as a complementary way to bound these exotic scenarios.

The paper is organized as follows. We begin in Sec. 2 by describing the formalism adopted for the non-standard electron-neutrino interactions and summarize the current bounds from a variety of experimental data. We then consider the process of relic neutrino decoupling in the presence of non-standard electron-neutrino interactions, giving first an estimate by using a semi-analytical approach in Sec. 3. Finally, in Sec. 4 we report the results of the full 
momentum-dependent numerical calculations for the neutrino spectra and the effect on the primordial ${ }^{4} \mathrm{He}$ yield and other cosmological observables. We present our conclusions in Sec. 5 .

\section{Non-standard neutrino-electron interactions}

The long-standing evidence of flavor change in atmospheric and solar neutrino experiments represents a strong indication of some new physics beyond the $\mathrm{SM}$ of fundamental interactions. For several years these experimental results have been interpreted in terms of neutrino masses (flavor oscillations) or by introducing new neutrino interactions. Recently, reactor and accelerator data have confirmed that neutrino masses are indeed the main mechanism explaining the atmospheric and solar anomalies, while the role of NSI can be only sub-leading. In any case, most extended particle physics models that account for neutrino masses naturally lead to new NSI, whose value strongly depends on the model. For instance, NSI may arise from the structure of the charged and neutral current weak interactions in seesaw-type extended models [7].

In the present analysis we will follow [3,5] and assume that new physics induces NSI only through the four fermion operators $(\bar{\nu} \nu)(\bar{f} f)$, where $f$ is a charged lepton or quark, but not new charged lepton physics at tree level. In particular, since we are interested in the decoupling process of relic neutrinos, we consider only the NSI related to electrons which, together with the standard weak interactions, are described by the effective Lagrangian

$$
\mathcal{L}_{\text {eff }}=\mathcal{L}_{\mathrm{SM}}+\sum_{\alpha, \beta} \mathcal{L}_{\mathrm{NSI}}^{\alpha \beta}
$$

which contains the four-fermion terms

$$
\begin{aligned}
& \mathcal{L}_{\mathrm{SM}}=-2 \sqrt{2} G_{F}\left\{\left(\bar{\nu}_{e} \gamma^{\mu} L \nu_{e}\right)\left(\bar{e} \gamma_{\mu} L e\right)+\sum_{P, \alpha} g_{P}\left(\bar{\nu}_{\alpha} \gamma^{\mu} L \nu_{\alpha}\right)\left(\bar{e} \gamma_{\mu} P e\right)\right\} \\
& \mathcal{L}_{\mathrm{NSI}}^{\alpha \beta}=-2 \sqrt{2} G_{F} \sum_{P} \varepsilon_{\alpha \beta}^{P}\left(\bar{\nu}_{\alpha} \gamma^{\mu} L \nu_{\beta}\right)\left(\bar{e} \gamma_{\mu} P e\right)
\end{aligned}
$$

for energies much smaller than the $Z$ boson mass, as in our case, with $G_{F}$ the Fermi constant and $P=L, R=\left(1 \mp \gamma_{5}\right) / 2$ the chiral projectors. Greek indices label lepton flavors $(\alpha, \beta=e, \mu, \tau)$ and the $Z$ couplings are $g_{L}=-\frac{1}{2}+\sin ^{2} \theta_{W}$ and $g_{R}=\sin ^{2} \theta_{W}$.

The NSI parameters $\varepsilon_{\alpha \beta}^{P}$ can induce a breaking of lepton universality ( $\alpha=$ $\beta$ ) or rather a flavor-changing contribution $(\alpha \neq \beta)$. Their values can be 
constrained by a variety of laboratory experiments, as discussed in $[3,5,6]$. In what follows we summarize the present bounds and refer the reader to the analyses mentioned above for all details. When considering the bounds on the $\varepsilon_{\alpha \beta}^{P}$ parameters, it is important to notice that they are usually obtained taking only one-at-a-time, or at most combining two of them (such as the pair $\left.\varepsilon_{e e}^{L, R}\right)$. This implies that the derived constraints are expected to be weaker but more robust if many NSI parameters are simultaneously included, since cancellations may occur.

A remark is in order. Since isospin-changing weak interactions converting neutrons into protons and vice versa are important for the BBN yields, one might wonder if it is legitimate to neglect the neutrino NSI with quarks. We argue that this approximation is well justified since, while relic neutrino distributions affect $n \leftrightarrow p$ processes in a crucial way, vice versa is not true. The highly suppressed density of baryons with respect to electromagnetic particles $\left(n_{b} / n_{\gamma} \simeq 6 \times 10^{-10}\right)$ allows one to neglect completely neutrino scattering on quarks for calculating the neutrino momentum spectrum. In addition, what contributes to the $n \leftrightarrow p$ rates are neutrino charged-current interactions, where possible non-standard terms are severely bounded by the accurate agreement of SM calculations for several processes with the data (e.g. leptonic and hadronic weak decays). For baryon thermalization, neutral current neutrino reactions are completely negligible with respect to electromagnetic interactions with $e^{ \pm}$and photons. Also note that any tiny non-standard effect in the weak rates is effectively taken into account by our prescription of rescaling the theoretically predicted neutron lifetime to the experimentally measured value (see [8] for details).

Finally, we comment on a possible role of exotic four-fermion neutrino-neutrino interactions of the kind parameterized in Eq. (3), reporting the bounds quoted in [9]. If only left-handed neutrinos are involved, the accurate measurement of the $Z$-boson width constrains the NSI coupling to be at most of the same strength of the neutral current ones in the SM, and thus we expect that they will have a sub-leading effect on our results (see Sec. 4). Instead, for NSI terms coupling left-handed to right-handed components, a stringent $\mathrm{BBN}$ bound of $\mathcal{O}\left(10^{-3}\right)$ applies, coming from the request that more than one neutrinoequivalent degree of freedom which was thermally populated in the early Universe is excluded. Finally, extra interactions coupling only right-handed states might be large, but this case is of no interest for the process of neutrino decoupling.

\subsection{Bounds from tree level processes}

- Neutrino scattering experiments 
The magnitude of the NSI parameters can be constrained from the analysis of data from neutrino-electron scattering experiments, which can probe the SM electroweak predictions with good precision (see e.g. [10]).

First, we focus on $\nu_{e}-e$ (data from the LSND experiment [11]) and $\bar{\nu}_{e}-e$ scattering (data from the Irvine [12] and MUNU [13] experiments). The total $\nu_{e}-e$ cross section including NSI is

$$
\begin{aligned}
\sigma\left(\nu_{e} e \rightarrow \nu e\right) & =\frac{2 G_{F}^{2} m_{e} E_{\nu}}{\pi}\left[\left(1+g_{L}+\varepsilon_{e e}^{L}\right)^{2}+\frac{1}{3}\left(g_{R}+\varepsilon_{e e}^{R}\right)^{2}\right. \\
& \left.+\sum_{\alpha \neq e}\left(\left|\varepsilon_{\alpha e}^{L}\right|^{2}+\frac{1}{3}\left|\varepsilon_{\alpha e}^{R}\right|^{2}\right)\right]
\end{aligned}
$$

while the LSND measurement is

$$
\sigma\left(\nu_{e} e \rightarrow \nu e\right)=(1.17 \pm 0.17) \frac{G_{F}^{2} m_{e} E_{\nu}}{\pi}
$$

In [5] this result was compared with the SM prediction $\sigma=1.0967 G_{F}^{2} m_{e} E_{\nu} / \pi$ including electroweak corrections and best fit value for $\sin ^{2} \theta_{W}$ - to obtain the following allowed regions around the SM value, assuming only one operator at a time

$$
\begin{aligned}
-0.07(-0.14) & <\varepsilon_{e e}^{L}<0.11(0.16) \\
-0.99(-1.14) & <\varepsilon_{e e}^{R}<0.53(0.67) \\
\left|\varepsilon_{e \tau}^{L}\right| & <0.4(0.5) \\
\left|\varepsilon_{e \tau}^{R}\right| & <0.7(0.9)
\end{aligned}
$$

at $90 \%$ CL (we added 99\% CL bounds in parentheses). For $\varepsilon_{e \mu}^{P}$ more severe bounds are obtained using radiative effects, see later. In general, the allowed region in the $\varepsilon_{e e}^{R}, \varepsilon_{e e}^{L}$ plane is an elliptic corona as given in Fig. 1, assuming $\varepsilon_{e \alpha}^{L, R}=0$ for $\alpha \neq e$. As shown in [6], with the addition of data from $\bar{\nu}_{e}-e$ scattering the allowed LSND region can be substantially reduced. Since one must exchange $g_{L} \leftrightarrow g_{R}$ with respect to Eq. (4) one obtains a perpendicular ellipse. The allowed regions for $\varepsilon_{e e}^{L, R}$ are shown in Fig. 1, while the one-parameter $90 \%$ CL bounds are now [6]

$$
\begin{aligned}
-0.05[-1.58] & <\varepsilon_{e e}^{L}<0.12[0.12] \\
-0.04[-0.61] & <\varepsilon_{e e}^{R}<0.14[0.15] \\
\left|\varepsilon_{e \tau}^{L}\right| & <0.44[0.85] \\
\left|\varepsilon_{e \tau}^{R}\right| & <0.27[0.38]
\end{aligned}
$$




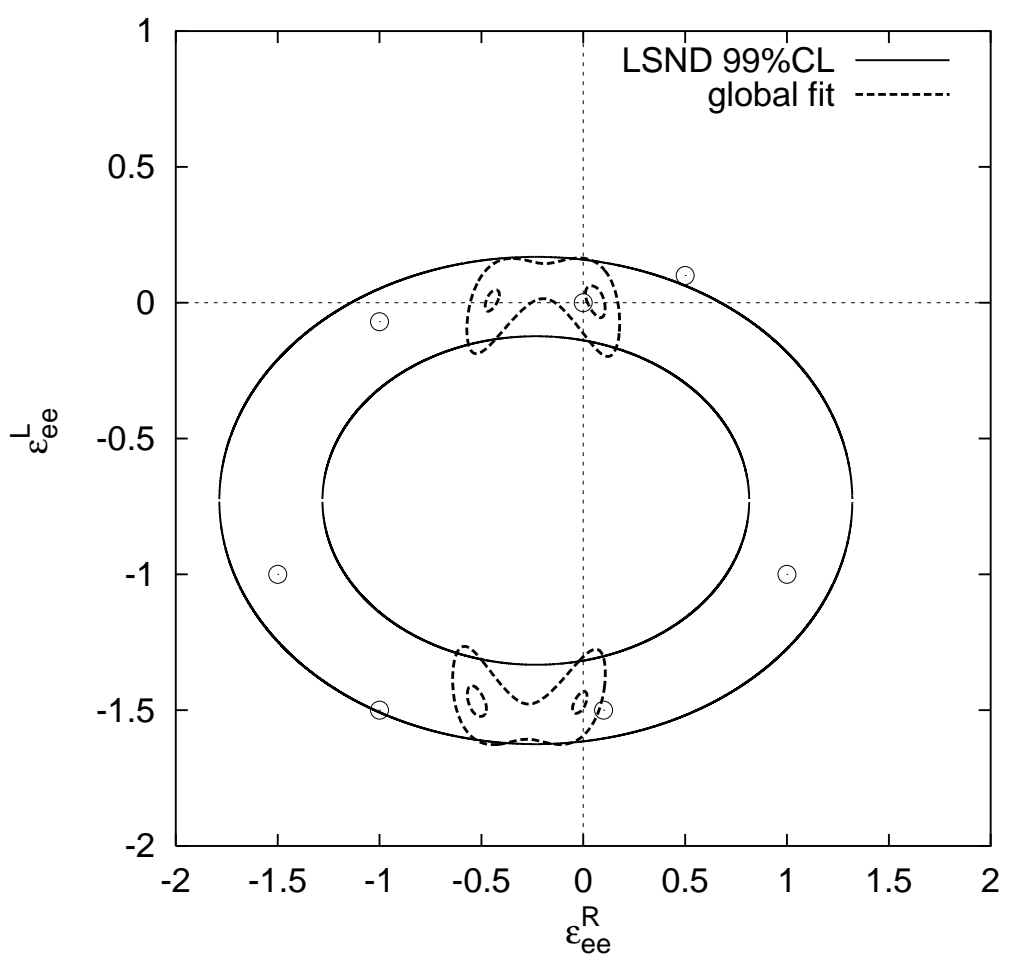

Fig. 1. The ellipse corresponds to the values of $\left(\varepsilon_{e e}^{L}, \varepsilon_{e e}^{R}\right)$ allowed at $99 \%$ CL by LSND data, while the dashed regions are those allowed at $1 \sigma$ and $99 \%$ CL when including data from antineutrino-electron experiments, as found in [6]. Here we assumed vanishing off-diagonal NSI parameters. We have calculated the full neutrino decoupling process for the indicated points (see Sec. 4).

When leaving the corresponding flavor-changing NSI parameters free, the bounds are relaxed to the numbers in square parentheses: note the significant reduction especially for negative $\varepsilon_{e e}^{R}$ values.

We also have data on $\nu_{\mu}-e$ scattering from the CHARM II collaboration [14]. The obtained results for the vector and axial vector $e-\nu_{\mu}$ couplings can be translated into the following 90\% CL bounds on NSI parameters (see e.g. [5])

$$
\begin{array}{r}
-0.025<\varepsilon_{\mu \mu}^{L}<0.03 \\
-0.027<\varepsilon_{\mu \mu}^{R}<0.03 \\
\left|\varepsilon_{\mu \tau}^{P}\right|<0.1
\end{array}
$$

which imply that the $\nu_{\mu}-e$ interactions must be very close to the SM predictions.

- LEP data on $e^{+} e^{-} \rightarrow \nu \bar{\nu} \gamma$

As pointed out in [3], neutrino NSI can be also constrained by measuring the 
$e^{+} e^{-} \rightarrow \nu \bar{\nu} \gamma$ cross section. This is actually the only way to get bounds on the $\varepsilon_{\tau \tau}^{L, R}$ parameters from laboratory data. The approximate limits for these parameters can be extracted from Fig. 3 of [4],

$$
\begin{aligned}
&-0.7 \lesssim \varepsilon_{\tau \tau}^{L} \lesssim 0.5 \\
&-0.5 \lesssim \varepsilon_{\tau \tau}^{R} \lesssim 0.6
\end{aligned}
$$

at $99 \% \mathrm{CL}$ when the other parameter is left free. For the $\nu_{e} e$ NSI the bounds are comparable to those obtained from neutrino-electron scattering.

\subsection{One loop effects}

The non-standard neutrino-electron interactions also lead to corrections at the one-loop level to processes such as the decays of the electroweak gauge bosons or lepton flavor violating decays of charged leptons. These corrections arise from effective non-renormalizable interactions and in principle their computation requires the knowledge of the complete theory leading to these effective terms in the low-energy regime. Nevertheless for $\Lambda \gg m_{W}$, where $\Lambda$ is the energy scale setting the limit of validity of the effective theory, the leading term is independent of the specific theory and has a logarithmic behavior, $\ln \left(\Lambda / m_{W}\right)$ [5]. Using conservatively $\ln \left(\Lambda / m_{W}\right) \simeq 1$, one gets the following $90 \%$ CL bounds from the decay rates of the electroweak gauge bosons

$$
\left|\varepsilon_{\tau \tau}^{L, R}\right| \lesssim 0.5
$$

which are of the same order of Eq. (11).

Similarly, from the strong experimental limit on the branching ratio $\operatorname{Br}\left(\mu^{-} \rightarrow\right.$ $\left.e^{-} e^{+} e^{-}\right)<10^{-12}$, it is possible to obtain a severe bound on the flavor changing parameter $(90 \% \mathrm{CL})$

$$
\left|\varepsilon_{e \mu}^{P}\right| \lesssim 5 \times 10^{-4}
$$

\subsection{Summary of bounds on NSI parameters}

After reviewing the bounds on NSI parameters, we conclude that they

- restrict the $\varepsilon_{e e}^{P}$ as shown in Fig. 1;

- force the $\varepsilon_{\mu \mu}^{P}$ to be very small and negligible for the purposes of the present paper;

- allow large $\varepsilon_{\tau \tau}^{P}$, almost of order unity, see (11); 
- as far as flavor changing parameters are concerned, allow large $\varepsilon_{e \tau}^{P}$, (7), moderate $\varepsilon_{\mu \tau}^{P},(10)$, and very tiny $\varepsilon_{e \mu}^{P}$, the latter being too small to have any impact on neutrino decoupling dynamics.

Again it is useful to remind the reader that the bounds on NSI parameters are relaxed when more than one parameter is allowed to vary simultaneously, as shown in [6].

As noted in [5], the leptonic measurement of $\sin ^{2} \theta_{W}$ at a neutrino factory will be sensitive to values of the $\nu_{e, \mu}-e$ NSI parameters of $\mathcal{O}\left(10^{-3}\right)$. Additional information on the NSI can be extracted from a consistency comparison of the results of solar neutrino experiments with those of an experiment detecting reactor neutrinos such as KamLAND. The former depend on the NSI parameters through the matter potential felt by solar neutrinos and/or via the neutrino neutral current detection. Instead, KamLAND data is essentially unaffected by the NSI. Ref. [5] found that the combination of SNO and KamLAND data ${ }^{1}$ will provide bounds on the $\varepsilon_{\tau \tau}^{P}$ comparable but slightly better to those in Eqs. (11) or (12). However, it has been noted in several works $[15,16,17,18,19]$ that while NSI are expected to play a subdominant role on the oscillations of atmospheric and solar neutrinos, there exist degenerate directions in the parameter space where large NSI are still allowed. Future data from short and medium baseline neutrino beams will help to resolve these degeneracies [20].

\section{Neutrino decoupling in presence of NSI}

\subsection{Delayed neutrino decoupling in the instantaneous limit}

In the early Universe, thermally produced neutrinos were in equilibrium with other particles down to temperatures of few $\mathrm{MeV}$, when weak interactions became ineffective and neutrino decoupled from the plasma. As a first approximation, the thermal Fermi-Dirac momentum spectrum is preserved after the freeze-out of weak interactions, since neutrinos decoupled when ultrarelativistic and both neutrino momenta and temperature redshift identically with the Universe expansion. A neutrino chemical potential $\mu_{\nu}$ would exist in the presence of a neutrino-antineutrino asymmetry, but it was shown in [21] that the stringent BBN bounds on $\mu_{\nu_{e}}$ (for an updated limit see e.g. [22]) apply to all flavors, since neutrino oscillations lead to flavor equilibrium before BBN. Thus we shall ignore a relic neutrino asymmetry in the following.

It proves useful to define the following dimensionless variables instead of time,

$\overline{1}$ The Borexino detector was considered in [4]. 
momenta and photon temperature

$$
x \equiv m R \quad y \equiv p R \quad z \equiv T_{\gamma} R
$$

where $m$ is an arbitrary mass scale which we choose as the electron mass $m_{e}$ and $R$ is the Universe scale factor. The function $R$ is normalized without loss of generality so that $R(t) \rightarrow 1 / T$ at large temperatures, $T$ being the common temperature of the particles in equilibrium far from any entropy-transfer process. With this choice, $R^{-1}$ can be identified with the temperature of neutrinos in the limit of instantaneous decoupling. After decoupling, neutrinos enter cosmological observables mainly via their energy density. Since they also remain relativistic for most of the cosmological evolution - down to a temperature of the order of their sub-eV mass scale - it is customary to parameterize their contribution to the radiation energy density $\rho_{\mathrm{R}}$ in terms of the effective number of neutrinos $N_{\text {eff }}[23,24]$

$$
N_{\mathrm{eff}} \equiv \frac{\rho_{\nu+X}}{\rho_{\nu}^{0}} \frac{\rho_{\gamma}^{0}}{\rho_{\gamma}}=\left(3+\sum_{\alpha}^{3} \delta_{\alpha}\right)\left(\frac{z_{0}}{z}\right)^{4}
$$

where $z_{0}(x)$ describes the photon to neutrino temperature ratio in the limit of instantaneous neutrino decoupling. For $x \gg 1$ the $e^{ \pm}$annihilation phase is over, and $z_{0} \rightarrow(11 / 4)^{1 / 3} \simeq 1.4010$. The energy densities $\rho_{\gamma}^{0}$ and $\rho_{\nu}^{0}$ refer respectively to the photon plasma and to a single neutrino species in the limit of instantaneous decoupling, while $\rho_{\gamma}$ is the actual energy content of the photon plasma and $\rho_{\nu+X}$ the total energy content of weakly interacting particles (including possible exotic contributions). The second equality in Eq. (15) follows when only the three active neutrinos contribute to $\rho_{\nu+X}$; eventually, the actual photon temperature evolution accounts for the second factor in Eq. (15) and the possible energy-density distortion in the $\alpha$-th neutrino flavor is given by $\delta_{\alpha} \equiv\left(\rho_{\nu_{\alpha}}-\rho_{\nu}^{0}\right) / \rho_{\nu}^{0}$. Note that from Eq. (15) it follows

$$
\rho_{\mathrm{R}}=\left(1+\frac{7}{8} z_{0}^{4} N_{\mathrm{eff}}\right) \rho_{\gamma}
$$

which, replacing $z_{0}$ with $(11 / 4)^{4 / 3}$, is often used in the literature to define $N_{\text {eff }}$ in the asymptotic limit $x \gg 1$. Clearly, well after $e^{ \pm}$annihilation, three thermally distributed neutrinos correspond to $N_{\text {eff }}=3$ in the instantaneous decoupling limit.

In order to estimate analytically how large would be the impact of NSI on the decoupling mechanism, let us discuss a simple toy-model, which we shall later compare with the results obtained solving the relevant kinetic equations. Consider the general case where $3-N^{\prime}$ neutrinos interact via standard weak processes, while the remaining $N^{\prime}$ have extra contributions from NSI which 
enhance the interaction rates over $e^{ \pm}$. In the instantaneous decoupling limit for both the neutrino species, the plasma can be described by a single additional parameter, the non-standard neutrino temperature $T^{\prime}$ or, equivalently, by $w \equiv$ $T^{\prime} R \geq 1$. From Eq. (15), one obtains

$$
N_{\mathrm{eff}}=\left[\left(3-N^{\prime}\right)+N^{\prime} w^{4}\right]\left(\frac{z_{0}}{z}\right)^{4}=\left[3+N^{\prime}\left(w^{4}-1\right)\right]\left(\frac{z_{0}}{z}\right)^{4}
$$

where the second step reflects the fact that, for a single NSI neutrino species, the distortion is given by $\delta^{\prime}=\left(w^{4}-1\right)$. If we denote with $x_{d} \equiv T_{d} R$ the epoch of the standard neutrino decoupling, and parameterize with $x_{d}^{\prime} \equiv T_{d}^{\prime} R$ the decoupling temperature of the non-standard species, we can easily calculate the functions $w(x)$ and $z(x)$ from the conservation of entropy per comoving volume,

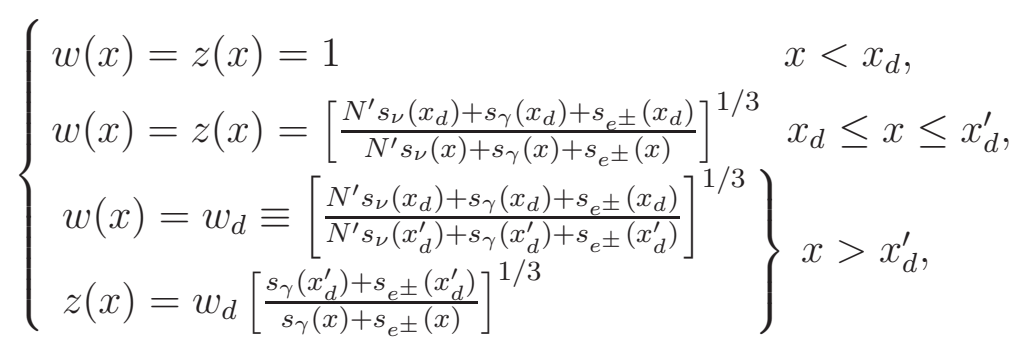

where $s_{\nu}, s_{\gamma}$ and $s_{e^{ \pm}}$are respectively the specific entropy of one $\nu-\bar{\nu}$ species, of the photons and of the $e^{ \pm}$.

In Fig. 2 we show the results for $N_{\text {eff }}$ in the instantaneous decoupling limit as function of the decoupling temperature $T_{d}^{\prime}$ and for various choices of $N^{\prime}$. As expected, $N_{\text {eff }}$ grows if neutrinos decouple at a smaller temperature and shows two regimes. For very small NSI contribution to the neutrino interaction rates we have $w_{d} \simeq 1$ and $N_{\text {eff }} \simeq 3$ since neutrinos decouple long before any $e^{ \pm}$annihilation. On the other hand, for $N^{\prime}$ neutrinos tightly coupled to the electromagnetic component down to very small temperatures when all $e^{ \pm}$pairs have already annihilated,

$$
z \simeq\left(\frac{22+7 N^{\prime}}{8+7 N^{\prime}}\right)^{1 / 3}, \quad w \simeq z
$$

Thus, in the tightly coupled limit, one finds $N_{\text {eff }} \rightarrow\{7.0,9.7,11.5\}$ for $N^{\prime}=$ $\{1,2,3\}$ respectively, as shown in Fig. 2 . Note that one obtains a contribution to the radiation energy density of order $N_{\text {eff }} \sim 4$ if neutrinos are kept in equilibrium down to temperatures of $0.2-0.3 \mathrm{MeV}$. 


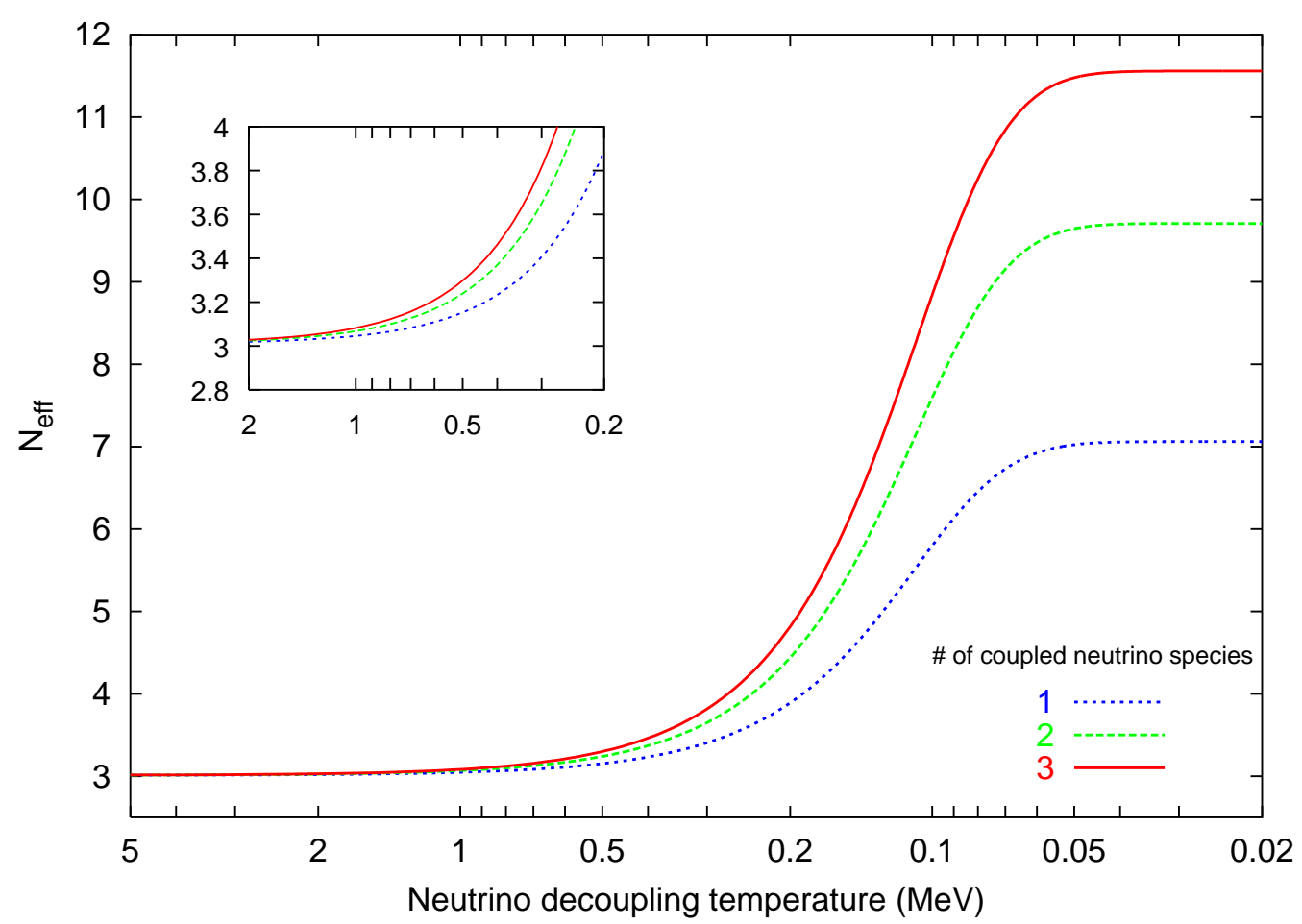

Fig. 2. Contribution to the radiation energy density parameterized in terms of $N_{\text {eff }}$, when 1, 2 or 3 neutrino species are coupled to the electromagnetic plasma until a given value of the decoupling temperature $T_{d}^{\prime}$.

\subsection{Decoupling temperature with NSI}

In order to dynamically predict the decoupling temperature $T_{d}^{\prime}$ when NSI are present, we have to solve the relevant kinetic equations taking into account the new couplings $\varepsilon$. For a first analytical estimate, we can compare the interaction rate $\Gamma$ with $e^{ \pm}$with the expansion rate of the Universe given by the Hubble parameter $H$. Since $H \propto T^{2}$, and $\Gamma \sim(1+\varepsilon)^{2} T^{5}$, the decoupling temperature at which $H=\Gamma$ decreases as $T_{d}^{\prime} \sim(1+\varepsilon)^{-2 / 3}$. As we have argued in the previous section, in order to produce changes of $\mathcal{O}(1)$ in $N_{\text {eff }}$ the decoupling temperature should be lowered down to $T_{d}^{\prime} \sim 0.2-0.3 \mathrm{MeV}$, i.e. should be one order of magnitude smaller than for ordinary neutrinos. This implies $\varepsilon \gtrsim 20$, which would largely exceed present laboratory bounds. We thus expect at most changes in $N_{\text {eff }}$ of $\mathcal{O}(0.01-0.1)$. To improve further our treatment, we can use an approximate solution of the kinetic equation for the neutrino distribution function $f_{\nu}(x, y)$,

$$
H x \frac{\partial f_{\nu}(x, y)}{\partial x}=I_{\nu}^{\mathrm{coll}}
$$



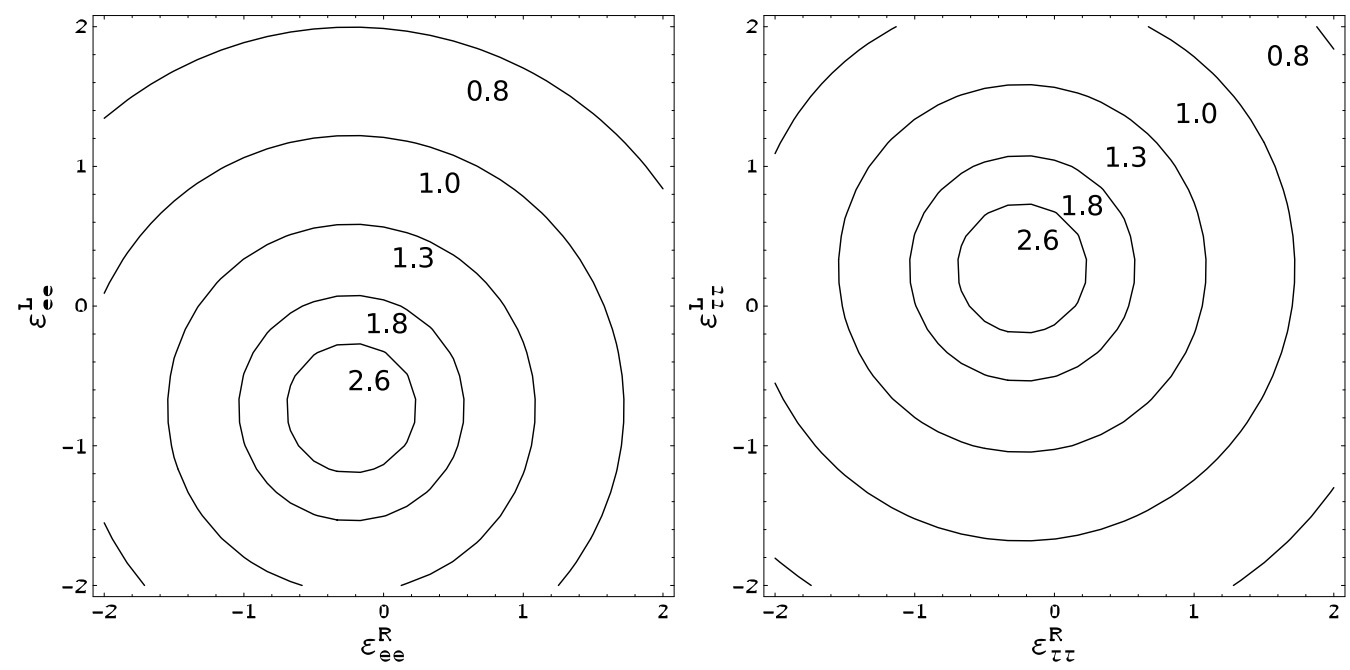

Fig. 3. Contours of equal neutrino decoupling temperature in $\mathrm{MeV}$ for different values of the parameters characterizing the $\nu_{e}-e$ and the $\nu_{\tau}-e$ diagonal NSI.

where $I_{\nu}^{\text {coll }}$ is the collision term that includes all relevant neutrino interaction processes. Following [25], we shall keep only the direct reaction term in the collision integral using the appropriate matrix elements for neutrino elastic scattering and the inverse annihilation $e^{+} e^{-} \rightarrow \bar{\nu} \nu$ in the relativistic limit $\left(m_{e} \rightarrow 0\right)$. Assuming that all particles that interact are close to equilibrium, the collision integral can be estimated in the Boltzmann approximation leading to

$$
H x \frac{\partial f_{\nu}}{f_{\nu} \partial x} \simeq-\frac{80 G_{F}^{2}\left(g_{L}^{2}+g_{R}^{2}\right) y}{3 \pi^{3} x^{5}}
$$

with $g_{L}^{2} \rightarrow\left(1+g_{L}\right)^{2}$ for the case of $\nu_{e}$. This equation can be easily integrated in $x$, leading to the values $T_{d}\left(\nu_{e}\right) \simeq 1.8 \mathrm{MeV}$ and $T_{d}\left(\nu_{\mu, \tau}\right) \simeq 3.1 \mathrm{MeV}$ for an average momentum of $\langle y\rangle=3.15$. This exercise can be repeated in the presence of neutrino-electron NSI in order to find their influence over the decoupling of neutrinos, adding $\varepsilon_{e e}^{L, R}$ or $\varepsilon_{\tau \tau}^{L, R}$ to the couplings $g_{L}$ and $g_{R}$. The calculated decoupling temperature is shown in Fig. 3 for the case of non-zero $\varepsilon_{e e}^{L, R}$ and $\varepsilon_{\tau \tau}^{L, R}$. In general, a significant increase of the NSI parameters from the SM prediction leads to a larger interaction of neutrinos with $e^{ \pm}$and thus to a lower decoupling temperature. However, for a region close to the pair of values $\left(\varepsilon^{L}, \varepsilon^{R}\right)$ that minimize the interaction by accidental cancellation with SM couplings, the decoupling temperature is significantly raised. Finally, one can relate the value of the decoupling temperature in presence of NSI with an estimate of the change in $N_{\text {eff }}$ following the instantaneous decoupling approximation previously described. The results are shown in Fig. 4. As already noticed, large modifications of $N_{\text {eff }}$ require that the neutrino-electron interactions are much larger than ordinary weak processes and are thus excluded by laboratory bounds. 

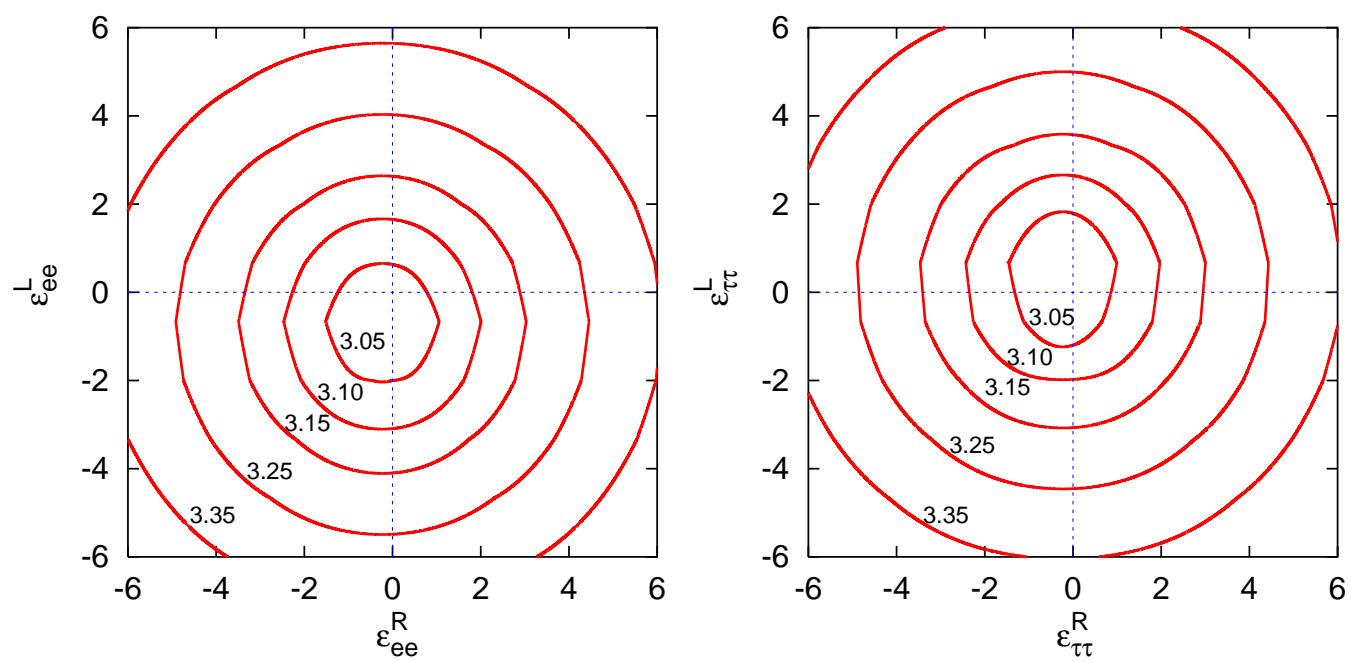

Fig. 4. Contours of equal $N_{\text {eff }}$ for different values of the NSI parameters as found in the instantaneous decoupling approximation.

Nonetheless, this study suggests that additional distortions in the neutrino spectra comparable to or larger than the ones predicted in the SM are possible, and it is still interesting to assess exactly how large these effects might be. However, the treatment followed till now assumes a series of approximations (such as $m_{e} \rightarrow 0$, no QED corrections to the plasma, no effect of the active neutrino oscillations, etc.) that in the next section we shall check using a fully numerical and momentum-dependent calculation.

\section{Numerical calculation: momentum-dependent kinetic equations}

\subsection{Method}

The instantaneous decoupling approximation does not take into account that neutrino-electron interactions are more efficient for larger neutrino energies. Thus one expects a priori that more energetic neutrinos will remain longer in thermal contact, leading in general to non-thermal distortions in the neutrino spectra and a slightly smaller increase of the comoving photon temperature $z$. This is actually the case for standard weak interactions, as noted in previous works (for early references, see [26] and the full list given in the review [25]).

A proper calculation of the process of non-instantaneous neutrino decoupling demands solving the momentum-dependent Boltzmann equations for the neutrino spectra, a set of integro-differential kinetic equations that are difficult to solve numerically. In the early 1990s several works [27,28,29] performed momentum-dependent calculations assuming some approximations, such as 
Boltzmann statistics for neutrinos, while the full numerical computation was later carried out in $[30,31,32,33]$. Finally, a further refinement involves the inclusion of finite temperature QED corrections to the electromagnetic plasma, $[34,35]$, while the last works also include the effect of flavor neutrino oscillations $[36,37]$.

Here we will follow our previous work [37]. In particular, we will describe the neutrino ensemble in the usual way by generalized occupation numbers, i.e. by $3 \times 3$ density matrices $\varrho$ for neutrinos and anti-neutrinos as described in [38,39], with elements $\varrho_{\alpha \beta}$ where $\alpha, \beta=e, \mu, \tau$. The diagonal elements correspond to the usual occupation numbers of the different flavors, while the off-diagonal terms are non-zero in the presence of neutrino mixing or flavor-changing NSI. The equations of motion for the density matrices relevant for our situation of interest in an expanding Universe are [38]

$$
i\left(\partial_{t}-H p \partial_{p}\right) \varrho_{p}=\left[\left(\frac{M^{2}}{2 p}-\frac{8 \sqrt{2} G_{\mathrm{F}} p}{3 m_{\mathrm{W}}^{2}} E\right), \varrho_{p}\right]+C\left[\varrho_{p}\right]
$$

where $m_{\mathrm{W}}$ is the $W$ boson mass. We use the notation $\varrho_{p}=\varrho(p, t)$ and $[\cdot, \cdot]$ denotes the commutator. The vacuum oscillation term is proportional to $M^{2}$, the mass-squared matrix in the flavor basis that is related to the diagonal one in the mass basis $\operatorname{diag}\left(m_{1}^{2}, m_{2}^{2}, m_{3}^{2}\right)$ via the neutrino mixing matrix $U$, which in turn depends on the neutrino mixing angles $\theta_{12}, \theta_{23}$ and $\theta_{13}$ (we assume CP conservation). From a global analysis of experimental data on flavor neutrino oscillations, the values of mixing parameters can be extracted. As a reference, we take the best-fit values from [1]

$$
\left(\frac{\Delta m_{21}^{2}}{10^{-5} \mathrm{eV}^{2}}, \frac{\Delta m_{31}^{2}}{10^{-3} \mathrm{eV}^{2}}, \mathrm{~s}_{12}^{2}, \mathrm{~s}_{23}^{2}, \mathrm{~s}_{13}^{2}\right)=(8.1,2.2,0.3,0.5,0)
$$

where $\mathrm{s}_{i j}^{2}=\sin ^{2} \theta_{i j}$ for $i, j=1,2,3$. In Ref. [37] we have shown that the results are not essentially modified for other values within the allowed regions, in particular for non-zero $\theta_{13}$ close to the experimental upper bound. Since we assume maximal $\theta_{23}$ and zero $\theta_{13}$ the spectra of $\nu_{\mu}$ and $\nu_{\tau}$ will be the same after decoupling.

The effects of the medium in Eq. (21) are contained in the collision term $C[\cdot]$ and in a refractive term that corresponds in Eq. (21) to the term proportional to the diagonal matrix $E$, that represents the energy densities of charged leptons. See [37] for a description of both terms. The kinetic equations (21) should be numerically solved along with the equation governing the evolution of the electromagnetic temperature during the process of $e^{ \pm}$annihilations. 
This can be found from the continuity equation for the total energy density $\rho$,

$$
\frac{d \rho}{d t}=-3 H(\rho+P),
$$

with $P$ the pressure, which can be cast into a first order differential equation for $z$ as function of $x$, see e.g. [35].

We have generalized the results of [37] calculating the full evolution of neutrino during decoupling in presence of non-standard interactions with electrons, which modify the equations in two different ways. First, the couplings are modified inserting the parameters $\varepsilon_{e e}^{L, R}, \varepsilon_{\tau \tau}^{L, R}$ or $\varepsilon_{e \tau}^{L, R}$, which implies a variation on the collisional term in Eq. (21). Second, neutrino refraction in the medium is modified by the NSI, which we took into account in the refractive term in Eq. (21)

$$
H_{\text {matt }}=-\frac{8 \sqrt{2} G_{F} p}{3 m_{W}^{2}} \rho_{e}\left(\begin{array}{ccc}
1+\varepsilon_{e e} & 0 & \varepsilon_{e \tau} \\
0 & 0 & 0 \\
\varepsilon_{e \tau} & 0 & \varepsilon_{\tau \tau}
\end{array}\right)
$$

where $\rho_{e}$ is the energy density of the background electrons and positrons and $\varepsilon_{\alpha \beta}=\varepsilon_{\alpha \beta}^{L}+\varepsilon_{\alpha \beta}^{R}$, since matter effects are sensitive only to the vector component of the interaction. The above equation includes only those NSI parameters that are not constrained to be smaller than $\mathcal{O}(0.1)$ (see Sec. 2), and thus represents the leading-order correction to the refractive index.

We have numerically solved the kinetic equations Eq. (21) using a discretization in a grid of 100 dimensionless neutrino momenta in the range $y_{i} \in$ $[0.02,20]$. We start to compute the evolution of the system at a value $x_{\mathrm{in}}$, when weak interactions were effective enough to keep neutrinos in equilibrium with the electromagnetic plasma but flavor oscillations are suppressed by medium effects. The system of equations is then solved from $x_{\text {in }}$ until a value of $x$ when both the neutrino distortions and the comoving photon temperature $z$ are frozen, approximately at $x_{\text {fin }} \simeq 35$. In some cases, we made the calculation for different initial conditions and concluded that the results are stable within per cent accuracy unless we start the numerical evaluation at $x_{\text {in }} \gtrsim 0.2$. For other technical details, we refer the reader to [37].

\subsection{Numerical results}

We have numerically calculated the evolution of the neutrino density matrix solving the system of Eqs. (21) and (23), during the full process of neutrino decoupling. Since a complete scan of all possible combinations of the NSI 


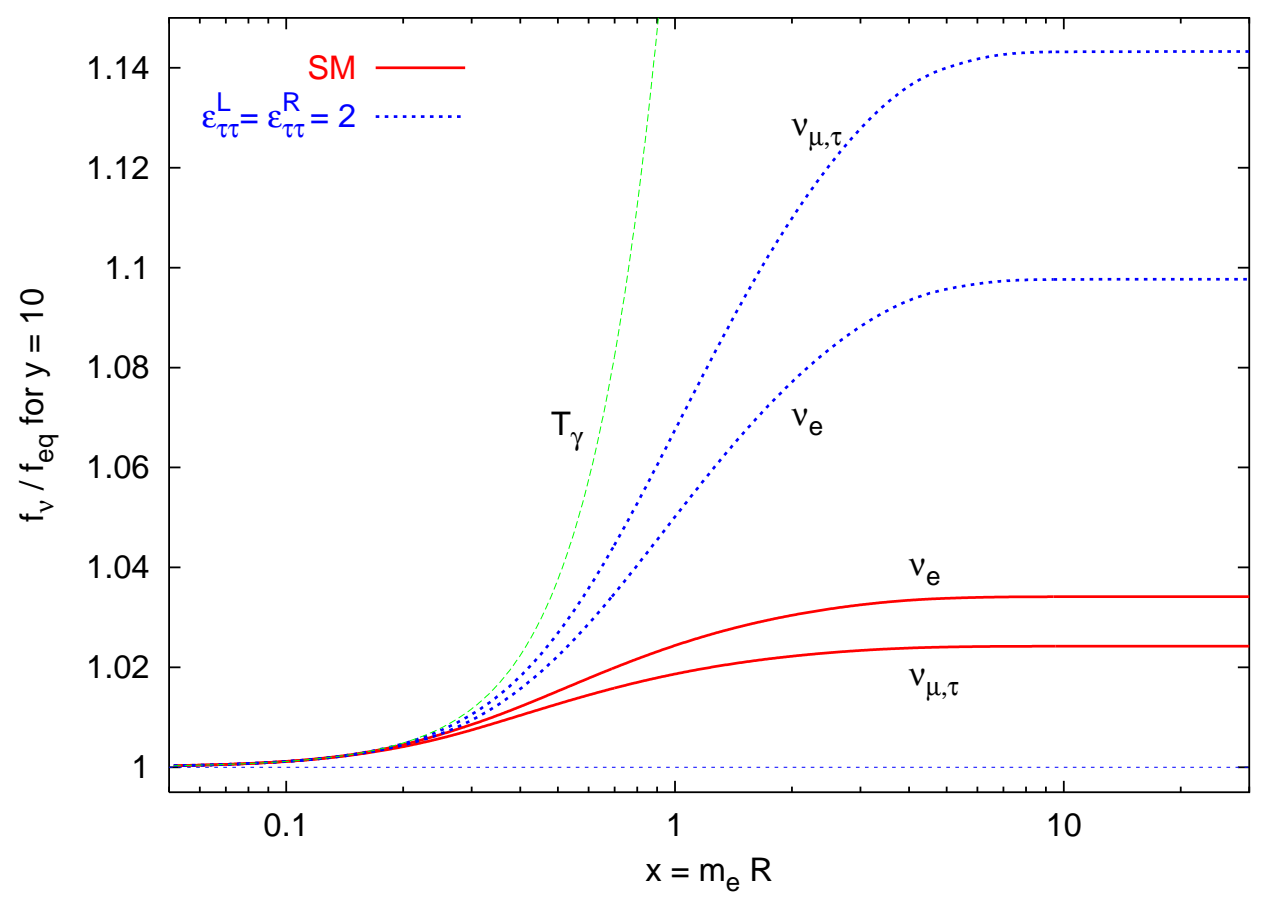

Fig. 5. Evolution of the distortion of the $\nu_{e}$ and $\nu_{\mu, \tau}$ spectra for a particular comoving momentum $(y=10)$ with standard weak interactions (solid line) and with $\nu_{\tau}-e$ NSI (dotted line). The line labelled with $T_{\gamma}$ corresponds to the distribution of a neutrino in full thermal contact with the electromagnetic plasma.

parameters can not be done, we have performed the calculation for a selection of values for the NSI parameters, representative of the regions allowed by present bounds (see Sec. 2).

As an example, in Fig. 5 we plot the distortion of the neutrino distribution as a function of $x$ for a particular neutrino momentum $(y=10)$, both in the case of SM weak interactions and in presence of large NSI between tau neutrinos and $e^{ \pm}$, corresponding to $\varepsilon_{\tau \tau}^{L}=\varepsilon_{\tau \tau}^{R}=2$. The behavior of $f_{\nu}$ has been previously described e.g. in $[31,33,37]$. At large temperatures or $x \lesssim 0.2$, neutrinos are in good thermal contact with $e^{ \pm}$and their distributions only change keeping an equilibrium shape with the photon temperature $[\exp (y / z(x))+1]^{-1}$ (the $T_{\gamma}$ line in the figure). In the intermediate region $0.2 \lesssim x \lesssim 4$, the standard weak interactions become less effective in a momentum-dependent way, leading to distortions in the neutrino spectra which are larger for $\nu_{e}$ 's than for the other flavors. Finally, at larger values of $x$ neutrino decoupling is complete and the distortions reach their asymptotic values. In the case of large $\nu_{\tau}-e$ NSI, neutrinos are kept in thermal contact with $e^{ \pm}$for a longer time, leading to larger distortions for all neutrino flavors, in particular for $\nu_{\mu, \tau}$ (although $\varepsilon_{e e}^{L, R}=\varepsilon_{\mu \mu}^{L, R}=0$, the electron and muon neutrinos also acquire significant distortions due to the effect of flavor oscillations). For the particular neutrino momentum in Fig. 5, if $\varepsilon_{\tau \tau}^{L}=\varepsilon_{\tau \tau}^{R}=2$ the final values of the distribution are $9.8 \%$ for the $\nu_{e}$ 's and $14.3 \%$ for the $\nu_{\mu, \tau}$ 's larger than in the limit of neutrino 
Table 1

Frozen values of $z_{\text {fin }}$, the neutrino energy densities distortion $\delta_{\alpha}, N_{\text {eff }}$ and $\Delta Y_{p}$ in presence of $\nu_{e}-e$ NSI.

\begin{tabular}{ccccccc}
\hline$\varepsilon_{e e}^{L}$ & $\varepsilon_{e e}^{R}$ & $z_{\text {fin }}$ & $\delta_{e}(\%)$ & $\delta_{\mu, \tau}(\%)$ & $N_{\text {eff }}$ & $\Delta Y_{p}$ \\
\hline 0 & 0 & 1.3978 & 0.73 & 0.52 & 3.046 & $2.1 \times 10^{-4}$ \\
\hline 0.1 & 0.5 & 1.3969 & 1.20 & 0.72 & 3.062 & $2.8 \times 10^{-4}$ \\
-1.0 & 1.0 & 1.3966 & 1.32 & 0.77 & 3.067 & $3.0 \times 10^{-4}$ \\
-1.5 & 0.1 & 1.3976 & 0.81 & 0.56 & 3.048 & $2.0 \times 10^{-4}$ \\
-1.5 & -1.0 & 1.3969 & 1.17 & 0.71 & 3.061 & $2.7 \times 10^{-4}$ \\
-1.0 & -1.5 & 1.3977 & 1.45 & 0.83 & 3.060 & $2.9 \times 10^{-4}$ \\
-0.07 & -1.0 & 1.3973 & 0.99 & 0.64 & 3.055 & $2.6 \times 10^{-4}$ \\
\hline 4.0 & 4.0 & 1.3812 & 9.47 & 3.83 & 3.357 & $11.7 \times 10^{-4}$ \\
\hline
\end{tabular}

decoupling before any $\mathrm{e}^{ \pm}$annihilation. For comparison, the corresponding values for standard weak interactions are $4.4 \%$ for the $\nu_{e}$ 's and $2 \%$ for the $\nu_{\mu, \tau}$ 's.

As in Ref. [37], we will summarize the results in terms of the following frozen values: dimensionless photon temperature $z_{\text {fin }}$, the fractional changes in the neutrino energy densities $\delta_{\alpha}$ and the asymptotic effective number of neutrinos $N_{\text {eff }}$ as defined in Eq. (15). We have considered four main sets of NSI parameters:

(1) Only $\varepsilon_{e e}^{L, R}$ : our results are summarized in Table 1 . We have calculated various combinations of the two $\nu_{e}-e$ NSI parameters, that correspond to the points shown in Fig. 1. For illustrative purposes, we considered the case with extremely large NSI $\varepsilon_{e e}^{L}=\varepsilon_{e e}^{R}=4$ (already excluded by laboratory bounds), where the distortions in the distribution functions of neutrinos lead to a significant change in $N_{\text {eff }}$.

(2) Only $\varepsilon_{\tau \tau}^{L, R}$ : our results are summarized in Table 2 for values of both parameters up to order unity in absolute value. Again, we also show one case with larger NSI $\varepsilon_{\tau \tau}^{L}=\varepsilon_{\tau \tau}^{R}=2$, disfavored by terrestrial experiments.

(3) Combinations of $\varepsilon_{e e}^{L, R}$ and $\varepsilon_{\tau \tau}^{L, R}$ : our results are summarized in Table 3 . Here we varied the values of the $\varepsilon_{e e}^{L, R}$ pair, while the $\nu_{\tau}-e$ NSI parameters were fixed to a value close to the current upper bound from laboratory experiments, $\varepsilon_{\tau \tau}^{L}=\varepsilon_{\tau \tau}^{R}=0.5$.

(4) Cases where the flavor-changing NSI parameters $\varepsilon_{e \tau}^{L, R}$ are non-zero, that for simplicity we consider real. We consider both cases with vanishing diagonal NSI and with all possible NSI parameters with the largest allowed values 
Table 2

Same as Table 1 but for $\nu_{\tau}-e$ NSI.

\begin{tabular}{ccccccc}
\hline$\varepsilon_{\tau \tau}^{L}$ & $\varepsilon_{\tau \tau}^{R}$ & $z_{\text {fin }}$ & $\delta_{e}(\%)$ & $\delta_{\mu, \tau}(\%)$ & $N_{\text {eff }}$ & $\Delta Y_{p}$ \\
\hline 0 & 0 & 1.3978 & 0.73 & 0.52 & 3.046 & $2.1 \times 10^{-4}$ \\
\hline 0.5 & 0.5 & 1.3970 & 0.90 & 0.79 & 3.059 & $4.0 \times 10^{-4}$ \\
1.0 & 1.0 & 1.3966 & 1.24 & 1.43 & 3.079 & $4.8 \times 10^{-4}$ \\
-1.0 & -1.0 & 1.3952 & 1.24 & 1.45 & 3.092 & $5.3 \times 10^{-4}$ \\
\hline 2.0 & 2.0 & 1.3911 & 2.04 & 2.96 & 3.168 & $10.0 \times 10^{-4}$ \\
\hline
\end{tabular}

Table 3

Same as Table 1 when both the $\nu_{e}-e$ and $\nu_{\tau}-e$ NSI are non-zero. In all the cases we have fixed $\varepsilon_{\tau \tau}^{L}=\varepsilon_{\tau \tau}^{R}=0.5$.

\begin{tabular}{ccccccc}
\hline$\varepsilon_{e e}^{L}$ & $\varepsilon_{e e}^{R}$ & $z_{\text {fin }}$ & $\delta_{e}(\%)$ & $\delta_{\mu, \tau}(\%)$ & $N_{\text {eff }}$ & $\Delta Y_{p}$ \\
\hline 0.1 & 0.5 & 1.3963 & 1.30 & 0.95 & 3.073 & $3.6 \times 10^{-4}$ \\
-1.0 & 1.0 & 1.3960 & 1.41 & 0.99 & 3.077 & $3.7 \times 10^{-4}$ \\
-1.5 & -1.0 & 1.3963 & 1.28 & 0.94 & 3.073 & $3.4 \times 10^{-4}$ \\
-1.0 & 0 & 1.3977 & 0.54 & 0.66 & 3.047 & $2.6 \times 10^{-4}$ \\
\hline
\end{tabular}

Table 4

Same as Table 1 when the flavor-changing NSI parameters $\varepsilon_{e \tau}^{L, R}$ are non-zero. The last two cases correspond to the results for all NSI parameters non-zero with values given by the bounds in Eqs. (8).

\begin{tabular}{ccccccccccc}
\hline$\varepsilon_{e e}^{L}$ & $\varepsilon_{e e}^{R}$ & $\varepsilon_{\tau \tau}^{L}$ & $\varepsilon_{\tau \tau}^{R}$ & $\varepsilon_{e \tau}^{L}$ & $\varepsilon_{e \tau}^{R}$ & $z_{\text {fin }}$ & $\delta_{e}(\%)$ & $\delta_{\mu, \tau}(\%)$ & $N_{\text {eff }}$ & $\Delta Y_{p}$ \\
\hline 0 & 0 & 0 & 0 & 0.4 & 0.7 & 1.3960 & 1.32 & 1.03 & 3.077 & $3.9 \times 10^{-4}$ \\
0 & 0 & 0 & 0 & 0.85 & 0.38 & 1.3956 & 1.46 & 1.16 & 3.085 & $4.4 \times 10^{-4}$ \\
0.12 & 0.15 & 0.5 & 0.5 & 0.85 & 0.38 & 1.3949 & 1.68 & 1.38 & 3.098 & $5.0 \times 10^{-4}$ \\
-0.61 & -1.58 & -0.5 & -0.5 & -0.85 & -0.38 & 1.3948 & 1.69 & 1.41 & 3.100 & $5.1 \times 10^{-4}$ \\
0.12 & -1.58 & -0.5 & 0.5 & -0.85 & 0.38 & 1.3937 & 2.21 & 1.66 & 3.120 & $6.0 \times 10^{-4}$ \\
\hline
\end{tabular}

according to Eqs. (8).

The last column $\left(\Delta Y_{p}\right)$ in Tables $1-4$ reports the variation in the mass yield of ${ }^{4}$ He synthesized during BBN, which predicts typically $Y_{p} \simeq 0.248$ for the baryon density $\omega_{b}=0.0223 \pm 0.0008$ favoured by CMB anisotropy studies [40]. Neutrinos affect the outcome of BBN in a flavor independent way through their contribution to the radiation energy density (change in $N_{\text {eff }}$ ). In addition, the 
role played by electron neutrinos and antineutrinos in the weak reactions that convert neutrons and protons is very important for fixing the primordial production of ${ }^{4} \mathrm{He}$, which is thus the nucleus mostly sensitive to non-standard physics in the neutrino sector. To predict quantitatively the change $\Delta Y_{p}$ induced by NSI, we take exactly into account the modified thermodinamical quantities, while treating perturbatively the change in the weak rates. This is justified given the relatively small effects we are considering. We closely follow the treatment given in [37], to which we refer the reader for further details.

The results are generally in agreement with the approximate ones predicted in Sec. 3 (see in particular Fig. 4), apart for minor differences due to the improved treatment. Also, as one naively expects, large $\varepsilon_{e e}^{L, R}$ enhance mostly the distortions in the electronic flavor, while large $\varepsilon_{\tau \tau}^{L, R}$ lead to larger distortions in the tau neutrino spectra. However, the differences between the spectra of the three neutrino flavors are reduced by the partial re-shuffling of the entropy transfer due to the effects of neutrino oscillations.

In general, we find that the presence of non-zero NSI enhances the transfer of entropy from $e^{ \pm}$to neutrinos, leading to values up to $N_{\text {eff }} \simeq 3.12$ when all NSI parameters are close to the boundaries of the allowed regions discussed in Sec. 2 , which is almost three times the departure of $N_{\text {eff }}$ from 3 that already exists for SM weak interactions (the first row in Table 1). We also obtained some significant departures of $N_{\text {eff }}$ from the standard value, such as $N_{\text {eff }} \simeq 3.36$ for $\varepsilon_{e e}^{L}=\varepsilon_{e e}^{R}=4$, but only for NSI parameters well beyond the allowed regions. On the other hand, for particular choices of the diagonal NSI parameters that minimize the $\nu-e$ interaction we find a reduction in the final value of the distortions or $N_{\text {eff }}$ with respect to the SM value, as expected from our discussion in Sec. 3.2.

Finally, we mentioned in Sec. 2 that neutrino-neutrino interactions are poorly constrained. In the case of interactions involving left-handed neutrinos only, they can still be twice as effective as in the SM, as reported in [9]. Since enhanced neutrino-neutrino interactions will redistribute the distortions more efficiently, they can modify the results described in this section. In order to check this, we doubled the intensity of the contributions of the neutrino-neutrino interactions to the collision integral and repeated the calculations for some of the cases considered above. We found that the results are basically unchanged, confirming the unimportant role played by neutrino-neutrino collisions in the process of neutrino decoupling. 


\section{Conclusions}

The process of relic neutrino decoupling in the early Universe is sensitive to the strength of the interactions between neutrinos and the plasma formed by electrons and positrons. If neutrinos were kept in longer thermal contact with them than in the standard case, they would share a larger fraction of the entropy release from $e^{ \pm}$annihilations. This would affect the predicted characteristics of the cosmic background of neutrinos $(\mathrm{C} \nu \mathrm{B})$, which in turn could modify the late evolution of the Universe and the bounds on neutrino properties from the analysis of cosmological observables.

In this paper we have considered how the decoupling of relic neutrinos is modified in presence of non-standard neutral current neutrino-electron interactions. First, we have provided a rough estimate of the size of NSI couplings needed to affect in a relevant way the properties of the $\mathrm{C} \nu \mathrm{B}$ and of related observables. We find that needed couplings are by far larger than the existing laboratory bounds, as also confirmed by a semi-analytical solution of the relevant kinetic equations. Thus, NSI can only play a minor role in shaping the $\mathrm{C} \nu \mathrm{B}$. However, NSI might still contribute with comparable or larger distortions in the neutrino spectra than predicted in the SM. In order to quantify these deviations, we have performed fully numerical and momentum-dependent calculations of the density-matrix equations relevant for neutrino evolution in the early Universe, including the effects of flavor neutrino oscillations.

Typically, we find that the enhancement in observables like $N_{\text {eff }}$, which measures the change in the radiation content, or the ${ }^{4} \mathrm{He}$ mass yield in the BBN can be up to three times larger than the ones found in the standard case [37], for values of all NSI parameters close to the limits placed by laboratory experiments. Nevertheless, even the variation of up to $0.2 \%$ that we found in the ${ }^{4} \mathrm{He}$ abundance is yet too small (about one order of magnitude) when compared to the observational error on $Y_{p}$, which is unfortunately dominated by systematics, and as such difficult to pin down in the near future. Instead, a value of $N_{\text {eff }} \simeq 3.12$, which is still possible within the present parameter space for NSI couplings, might even be barely detectable. Indeed, it has been shown that the CMB satellite PLANCK will soon provide temperature and polarization data that will probably measure the value of $N_{\text {eff }}$ with an uncertainty of order $\sigma\left(N_{\text {eff }}\right) \simeq 0.2[41,42]$ (for a previous, more optimistic forecast see [43]), or better when combined with data from a large galaxy redshift survey such as SDSS [44]. Future CMB missions may reach the sensitivity of $0.04-0.05$ needed to test the standard scenario [42], and might achieve a $2 \sigma$ hint for $N_{\text {eff }} \simeq 3.12$ and eventually test the effect of large neutrino-electron NSI.

However, barring some extreme cases, we conclude that the prediction of $N_{\text {eff }} \simeq 3$ within a few $\%$ is quite robust even when taking into account neutrino- 
electron NSI of the four-fermion type. Thus, their existence can not modify in a significant way the bounds on neutrino properties from cosmological observables, in particular on their masses, as recently reviewed in [45]. Since it is likely that future experiments may narrow further the allowed range of NSI couplings, their role in a cosmological context will be even smaller, at most a sub-leading correction to the standard prediction of the $\mathrm{C} \nu \mathrm{B}$ properties. Turning the argument around, however, one can conclude that a significant deviation of $N_{\text {eff }}$ from 3 may require major revisions of the cosmological model, like the introduction of new relativistic relics and/or of an exotic thermal history, or both (see e.g. $[46,47])$.

\section{Acknowledgments}

We thank Omar Miranda and Célio A. de Moura for fruitful discussions. This work was supported by a Spanish-Italian AI, the Spanish grants FPA200501269 and GV/05/017 of Generalitat Valenciana, as well as a MEC-INFN agreement. SP was supported by a Ramón y Cajal contract of MEC. PS acknowledges the support by the Deutsche Forschungsgemeinschaft under grant SFB 375 and by the European Network of Theoretical Astroparticle Physics ILIAS/N6 under contract number RII3-CT-2004-506222.

\section{References}

[1] M. Maltoni, T. Schwetz, M.A. Tórtola and J.W.F. Valle, New J. Phys. 6 (2004) 122 hep-ph/0405172.

[2] G.L. Fogli, E. Lisi, A. Marrone and A. Palazzo, Prog. Part. Nucl. Phys. 57 (2006) 742 hep-ph/0506083.

[3] Z. Berezhiani and A. Rossi, Phys. Lett. B 535 (2002) 207 hep-ph/0111137.

[4] Z. Berezhiani, R.S. Raghavan and A. Rossi, Nucl. Phys. B 638 (2002) 62 hep-ph/0111138.

[5] S. Davidson, C. Peña-Garay, N. Rius and A. Santamaria, JHEP 0303 (2003) 011 hep-ph/0302093.

[6] J. Barranco, O.G. Miranda, C.A. Moura and J.W.F. Valle, Phys. Rev. D 73 (2006) 113001 hep-ph/0512195.

[7] J. Schechter and J.W.F. Valle, Phys. Rev. D 22 (1980) 2227.

[8] P.D. Serpico et al., J. Cosmol. Astrop. Phys. 0412 (2004) 010 astro-ph/0408076. 
[9] M.S. Bilenky and A. Santamaria, hep-ph/9908272,

[10] A. de Gouvêa and J. Jenkins, Phys. Rev. D 74 (2006) 033004 hep-ph/0603036.

[11] L.B. Auerbach et al. [LSND Coll.], Phys. Rev. D 63 (2001) 112001 hep-ex/0101039.

[12] F. Reines, H.S. Gurr and H.W. Sobel, Phys. Rev. Lett. 37 (1976) 315.

[13] Z. Daraktchieva et al. [MUNU Coll.], Phys. Lett. B 564 (2003) 190 hep-ex/0304011.

[14] P. Vilain et al. [CHARM-II Coll.], Phys. Lett. B 335 (1994) 246.

[15] A. Friedland, C. Lunardini and C. Peña-Garay, Phys. Lett. B 594 (2004) 347 hep-ph/0402266.

[16] M.M. Guzzo, P.C. de Holanda and O.L.G. Peres, Phys. Lett. B 591 (2004) 1 hep-ph/0403134.

[17] O.G. Miranda, M.A. Tórtola and J.W.F. Valle, hep-ph/0406280

[18] A. Friedland, C. Lunardini and M. Maltoni, Phys. Rev. D 70 (2004) 111301 hep-ph/0408264.

[19] A. Friedland and C. Lunardini, Phys. Rev. D $72 \quad$ (2005) 053009 hep-ph/0506143.

[20] A. Friedland and C. Lunardini, hep-ph/0606101.

[21] A.D. Dolgov et al., Nucl. Phys. B 632 (2002) 363 hep-ph/0201287.

[22] P.D. Serpico and G.G. Raffelt, Phys. Rev. D $71 \quad$ (2005) 127301 astro-ph/0506162.

[23] V.F. Shvartsman, Pisma Zh. Eksp. Teor. Fiz. 9 (1969) 315 [JETP Lett. 9 (1969) 184].

[24] G. Steigman, D.N. Schramm and J.R. Gunn, Phys. Lett. B 66 (1977) 202.

[25] A.D. Dolgov, Phys. Rep. 370 (2002) 333 hep-ph/0202122.

[26] D.A. Dicus et al., Phys. Rev. D 26 (1982) 2694.

[27] S. Dodelson and M.S. Turner, Phys. Rev. D 46 (1992) 3372.

[28] A.D. Dolgov and M. Fukugita, Phys. Rev. D 46 (1992) 5378.

[29] B.D. Fields, S. Dodelson and M.S. Turner, Phys. Rev. D 47 (1993) 4309 astro-ph/9210007.

[30] S. Hannestad and J. Madsen, Phys. Rev. D 52 (1995) 1764 astro-ph/9506015.

[31] A.D. Dolgov, S.H. Hansen and D.V. Semikoz, Nucl. Phys. B 503 (1997) 426 hep-ph/9703315. 
[32] A.D. Dolgov, S.H. Hansen and D.V. Semikoz, Nucl. Phys. B 543 (1999) 269 hep-ph/9805467.

[33] S. Esposito et al., Nucl. Phys. B 590 (2000) 539 astro-ph/0005573.

[34] N. Fornengo, C.W. Kim and J. Song, Phys. Rev. D 56 (1997) 5123 hep-ph/9702324.

[35] G. Mangano, G. Miele, S. Pastor and M. Peloso, Phys. Lett. B 534 (2002) 8 astro-ph/0111408.

[36] S. Hannestad, Phys. Rev. D 65 (2002) 083006 astro-ph/0111423.

[37] G. Mangano et al., Nucl. Phys. B 729 (2005) 221 hep-ph/0506164.

[38] G. Sigl and G. Raffelt, Nucl. Phys. B 406 (1993) 423.

[39] B.H. McKellar and M.J. Thomson, Phys. Rev. D 49 (1994) 2710.

[40] D.N. Spergel et al., astro-ph/0603449.

[41] R. Bowen et al., MNRAS 334 (2002) 760 astro-ph/0110636.

[42] S. Bashinsky and U. Seljak, Phys. Rev. D 69 (2004) 083002 astro-ph/0310198.

[43] R.E. Lopez, S. Dodelson, A. Heckler and M.S. Turner, Phys. Rev. Lett. 82 (1999) 3952 astro-ph/9803095.

[44] J. Lesgourgues, S. Pastor and L. Perotto, Phys. Rev. D 70 (2004) 045016 hep-ph/0403296.

[45] J. Lesgourgues and S. Pastor, Phys. Rept. 429 (2006) 307 astro-ph/0603494.

[46] P.D. Serpico and G.G. Raffelt, Phys. Rev. D $70 \quad$ (2004) 043526 astro-ph/0403417.

[47] A. Cuoco, J. Lesgourgues, G. Mangano and S. Pastor, Phys. Rev. D 71 (2005) 123501 astro-ph/0502465. 